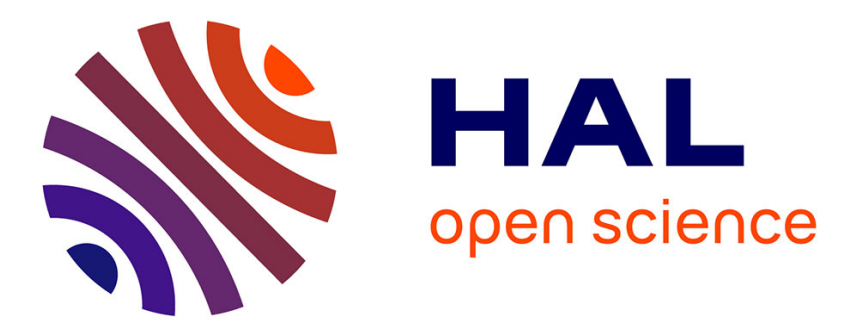

\title{
A mathematical study of the hematopoiesis process with applications to chronic myelogenous leukemia
}

Mostafa Adimy, Fabien Crauste, Shigui Ruan

\section{To cite this version:}

Mostafa Adimy, Fabien Crauste, Shigui Ruan. A mathematical study of the hematopoiesis process with applications to chronic myelogenous leukemia. SIAM Journal on Applied Mathematics, 2005, 65 (4), pp.1328-1352. 10.1137/040604698 . hal-00375977

\section{HAL Id: hal-00375977 \\ https://hal.science/hal-00375977}

Submitted on 16 Apr 2009

HAL is a multi-disciplinary open access archive for the deposit and dissemination of scientific research documents, whether they are published or not. The documents may come from teaching and research institutions in France or abroad, or from public or private research centers.
L'archive ouverte pluridisciplinaire HAL, est destinée au dépôt et à la diffusion de documents scientifiques de niveau recherche, publiés ou non, émanant des établissements d'enseignement et de recherche français ou étrangers, des laboratoires publics ou privés. 


\title{
A Mathematical Study of the Hematopoiesis Process with Applications to Chronic Myelogenous Leukemia
}

\author{
Mostafa Adimy ${ }^{\dagger}, \quad$ Fabien Crauste ${ }^{\dagger}$ and Shigui Ruan* \\ Year 2004 \\ †Laboratoire de Mathématiques Appliquées, FRE 2570, \\ Université de Pau et des Pays de l'Adour, \\ Avenue de l'université, 64000 Pau, France. \\ E-mail: mostafa.adimy@univ-pau.fr,fabien.crauste@univ-pau.fr \\ * Department of Mathematics, University of Miami, \\ P. O. Box 249085, Coral Gables, FL 33124-4250, USA. \\ E-mail: ruan@math.miami.edu
}

\begin{abstract}
This paper is devoted to the analysis of a mathematical model of blood cells production in the bone marrow (hematopoiesis). The model is a system of two age-structured partial differential equations. Integrating these equations over the age, we obtain a system of two nonlinear differential equations with distributed time delay corresponding to the cell cycle duration. This system describes the evolution of the total cell populations. By constructing a Lyapunov functional, it is shown that the trivial equilibrium is globally asymptotically stable if it is the only equilibrium. It is also shown that the nontrivial equilibrium, the most biologically meaningful one, can become unstable via a Hopf bifurcation. Numerical simulations are carried out to illustrate the analytical results. The study maybe helpful in understanding the connection between the relatively short cell cycle durations and the relatively long periods of peripheral cell oscillations in some periodic hematological diseases.
\end{abstract}

Keywords: Blood cells, hematopoiesis, differential equations, distributed delay, asymptotic stability, Lyapunov functional, Hopf bifurcation.

\section{Introduction}

Cellular population models have been investigated intensively since the 1960's (see, for example, Trucco [33, 34], Nooney [25], Rubinow 28] and Rubinow and Lebowitz [29]) and still

\footnotetext{
* Research was partially supported by the NSERC of Canada and the College of Arts and Sciences at the University of Miami.
} 
interest a lot of researchers. This interest is greatly motivated, on one hand, by the medical applications and, on the other hand, by the biological phenomena (such as oscillations, bifurcations, traveling waves or chaos) observed in these models and, generally speaking, in the living world (Mackey and Glass [19], Mackey and Milton [20]).

Hematopoiesis is the process by which primitive stem cells proliferate and differentiate to produce mature blood cells. It is driven by highly coordinated patterns of gene expression under the influence of growth factors and hormones. The regulation of hematopoiesis is about the formation of blood cell elements in the body. White and red blood cells and platelets are produced in the bone marrow from where they enter the blood stream. The principal factor stimulating red blood cell production is the hormone produced in the kidney, called erythropoietin. About $90 \%$ of the erythropoietin is secreted by renal tubular epithelial cells when blood is unable to deliver sufficient oxygen. When the level of oxygen in the blood decreases this leads to a release of a substance, which in turn causes an increase in the release of the blood elements from the marrow. There is a feedback from the blood to the bone marrow. Abnormalities in the feedback are considered as major suspects in causing

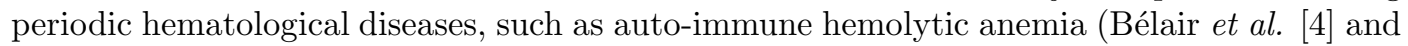
Mahaffy et al. 23]), cyclical neutropenia (Haurie et al. 14), chronic myelogenous leukemia (Fowler and Mackey [12] and Pujo-Menjouet et al. [26]), etc.

Cell biologists classified stem cells as proliferating cells and resting cells (also called $G_{0^{-}}$ cells) (see Mackey [16, 17]). Proliferating cells are committed to undergo mitosis a certain time after their entrance into the proliferating phase. Mackey supposed that this time of cytokinesis is constant, that is, it is the same for all cells. Most of committed stem cells are in the proliferating phase. The $G_{0}$-phase, whose existence is known due to the works of Burns and Tannock [8], is a quiescent stage in the cellular development. However, it is usually believed that $95 \%$ of pluripotent stem cells are in the resting phase. Resting cells can exit randomly to either entry into the proliferating phase or be irremediably lost. Proliferating cells can also be lost by apoptosis (programmed cell death).

The model of Mackey 16] has been numerically studied by Mackey and Rey 21 and Crabb et al. [9]. Computer simulations showed that there exist strange behaviors of the stem cell population, such as oscillations and bifurcations. Recently, Pujo-Menjouet and Mackey 27] proved the existence of a Hopf bifurcation which causes periodic chronic myelogenous leukemia and showed the great dependence of the model on the parameters.

In this paper, based on the model of Mackey [16], we propose a more general model of hematopoiesis. We take into account the fact that a cell cycle has two phases, that is, stem cells in process are either in a resting phase or actively proliferating. However, we do not suppose that all cells divide at the same age, because this hypothesis is not biologically reasonable. For example, it is believed that pluripotent stem cells divide faster than committed stem cells, which are more mature cells. There are strong evidences (see Bradford et al. [7] ) that indicate that the age of cytokinesis $\tau$ is distributed on an interval $[\underline{\tau}, \bar{\tau}]$ with $\underline{\tau} \geq 0$. Hence, we shall assume that $\tau$ is distributed with a density $f$ supported on an interval $[\underline{\tau}, \bar{\tau}]$, with $0 \leq \underline{\tau}<\bar{\tau}<+\infty$. The resulting model is a system of two differential equations with distributed delay. A simpler model, dealing with the pluripotent stem cell population behavior, has been studied by Adimy et al. [1].

Some results about stability of differential equations with distributed delay can be mentioned. In [6], Boese studied the stability of a differential equation with gamma-distributed delay. Gamma distributions have the property to simplify the nature of the delay and this situation is close to the one with discrete delay. Anderson [2, 3] showed stability results linked to the different moments (especially the expectation and the variance) of the distribution. Kuang 15] also obtained general stability results for systems of delay differential equations. More recently, sufficient conditions for the stability of delay differential equations 
with distributed delay have been obtained by Bernard et al. [5]. They used some properties of the distribution to prove these results. However, in all these works, the authors focused on sufficient conditions for the stability, there is no necessary condition in these studies, and these results are not applicable directly to the model considered in this paper.

This paper is organized as follows: in Section 2, we present the model and establish boundedness properties of the solutions. In Section 3, we study the asymptotic stability of the equilibria. We give conditions for the trivial equilibrium to be globally asymptotically stable in Section 3.1 and investigate the stability of the nontrivial equilibrium in Section 3.2. In Section 4 , we show that a local Hopf bifurcation occurs in our model. In Section 5 , numerical simulations are performed to demonstrate that our results can be used to explain the long period oscillations observed in chronic myelogenous leukemia.

\section{The hematopoiesis process: presentation of the model}

Denote by $r(t, a)$ and $p(t, a)$ the population densities of resting an proliferating cells, respectively, which have spent a time $a \geq 0$ in their phase at time $t \geq 0$. Resting cells can either be lost randomly at a rate $\delta \geq 0$, which takes into account the cellular differentiation, or entry into the proliferating phase at a rate $\beta$. Proliferating cells can be lost by apoptosis (a programmed cell death) at a rate $\gamma \geq 0$ and, at mitosis, cells with age $a$ divide in two daughter cells (which immediately enter the $G_{0}$-phase) with a rate $g(a)$.

The function $g:[0, \bar{\tau}) \rightarrow \mathbb{R}^{+}$satisfies $g(a)=0$ if $a<\underline{\tau}$ with $0 \leq \underline{\tau}<\bar{\tau}<+\infty$. Moreover, it is assumed to be piecewisely continuous such that $\int_{\tau}^{\bar{\tau}} g(a) d a=+\infty$. The later assumption describes the fact that cells which did not die have to divide before they reach the maximal age $\bar{\tau}$.

The nature of the trigger signal for introduction in the proliferating phase is not clear. However, the work of Sachs [30] shows that we can reasonably think that it strongly depends on the entire resting cell population, that is $\beta=\beta(x(t))$, with

$$
x(t)=\int_{0}^{+\infty} r(t, a) d a, \quad t \geq 0 .
$$

The function $\beta$ is supposed to be continuous and positive. Furthermore, from a reasonable biological point of view, we assume that $\beta$ is decreasing with $\lim _{x \rightarrow+\infty} \beta(x)=0$. This describes the fact that the rate of re-entry into the proliferating compartment is a decreasing function of the $G_{0}$-phase population.

Usually, it is believed that the function $\beta$ is a monotone decreasing Hill function (see Mackey 16), given by

$$
\beta(x)=\beta_{0} \frac{\theta^{n}}{\theta^{n}+x^{n}}, \quad x \geq 0,
$$

with $\beta_{0}>0, \theta \geq 0$ and $n>0$. $\beta_{0}$ is the maximal rate of re-entry in the proliferating phase, $\theta$ is the number of resting cells at which $\beta$ has its maximum rate of change with respect to the resting phase population, and $n$ describes the sensitivity of the reintroduction rate with changes in the population.

The above parameters values are usually chosen (see Mackey [16]) to be

$$
\delta=0.05 \text { day }^{-1}, \quad \gamma=0.2 \text { day }^{-1}, \quad \beta_{0}=1.77 \text { day }^{-1} \quad \text { and } \quad n=3 .
$$

Although an usual value of $\theta$ is $\theta=1.62 \times 10^{8}$ cells $/ \mathrm{kg}$, it can be normalized without loss of generality when one makes a qualitative analysis of the population. 
Then $r(t, a)$ and $p(t, a)$ satisfy the system of partial differential equations

$$
\begin{array}{lll}
\frac{\partial r}{\partial t}+\frac{\partial r}{\partial a}=-(\delta+\beta(x(t))) r, & & a>0, t>0, \\
\frac{\partial p}{\partial t}+\frac{\partial p}{\partial a}=-(\gamma+g(a)) p, & & 0<a<\bar{\tau}, t>0,
\end{array}
$$

with

$$
r(0, a)=\nu(a), a \geq 0, \quad p(0, a)=\Gamma(a), a \in[0, \bar{\tau}] .
$$

The functions $\nu=\nu(a)$ and $\Gamma=\Gamma(a)$ give the population densities of cells which have spent a time $a$ in the resting and proliferating phase, respectively, at time $t=0$; that is the initial populations of cells with age $a$ in each phase.

The boundary conditions of system (3)-(14), which describe the cellular flux between the two phases, are given by

$$
\left\{\begin{aligned}
r(t, 0) & =2 \int_{\underline{\tau}}^{\bar{\tau}} g(\tau) p(t, \tau) d \tau \\
p(t, 0) & =\beta(x(t)) x(t)
\end{aligned}\right.
$$

Moreover, we suppose that $\lim _{a \rightarrow+\infty} r(t, a)=0$ and $\lim _{a \rightarrow \bar{\tau}} p(t, a)=0$.

Let $y(t)$ denote the total population density of proliferating cells at time $t$; then

$$
y(t)=\int_{0}^{\bar{\tau}} p(t, a) d a, \quad t \geq 0 .
$$

Thus, integrating (3) and (4) with respect to the age variable, we obtain

$$
\begin{aligned}
& \frac{d x}{d t}=-(\delta+\beta(x(t))) x(t)+2 \int_{\underline{\tau}}^{\bar{\tau}} g(\tau) p(t, \tau) d \tau \\
& \frac{d y}{d t}=-\gamma y(t)+\beta(x(t)) x(t)-\int_{\underline{\tau}}^{\bar{\tau}} g(\tau) p(t, \tau) d \tau
\end{aligned}
$$

We define a function $G$ by

$$
G(t, a)= \begin{cases}g(a) \exp \left(-\int_{a-t}^{a} g(s) d s\right), & \text { if } t<a, \\ g(a) \exp \left(-\int_{0}^{a} g(s) d s\right), & \text { if } a<t .\end{cases}
$$

Set

$$
f(\tau):=g(\tau) \exp \left(-\int_{0}^{\tau} g(s) d s\right), \quad \tau>0 .
$$

One can check that $f$ is a density function, supported on $[\underline{\tau}, \bar{\tau}]$, and $f$ represents the density of division of proliferating cells. In particularly, $\int_{\underline{\tau}}^{\bar{\tau}} f(\tau) d \tau=1$.

Using the method of characteristics to determine $p(t, a)$, we deduce, from (5)-(6), that 
the process of hematopoiesis is described by the following system:

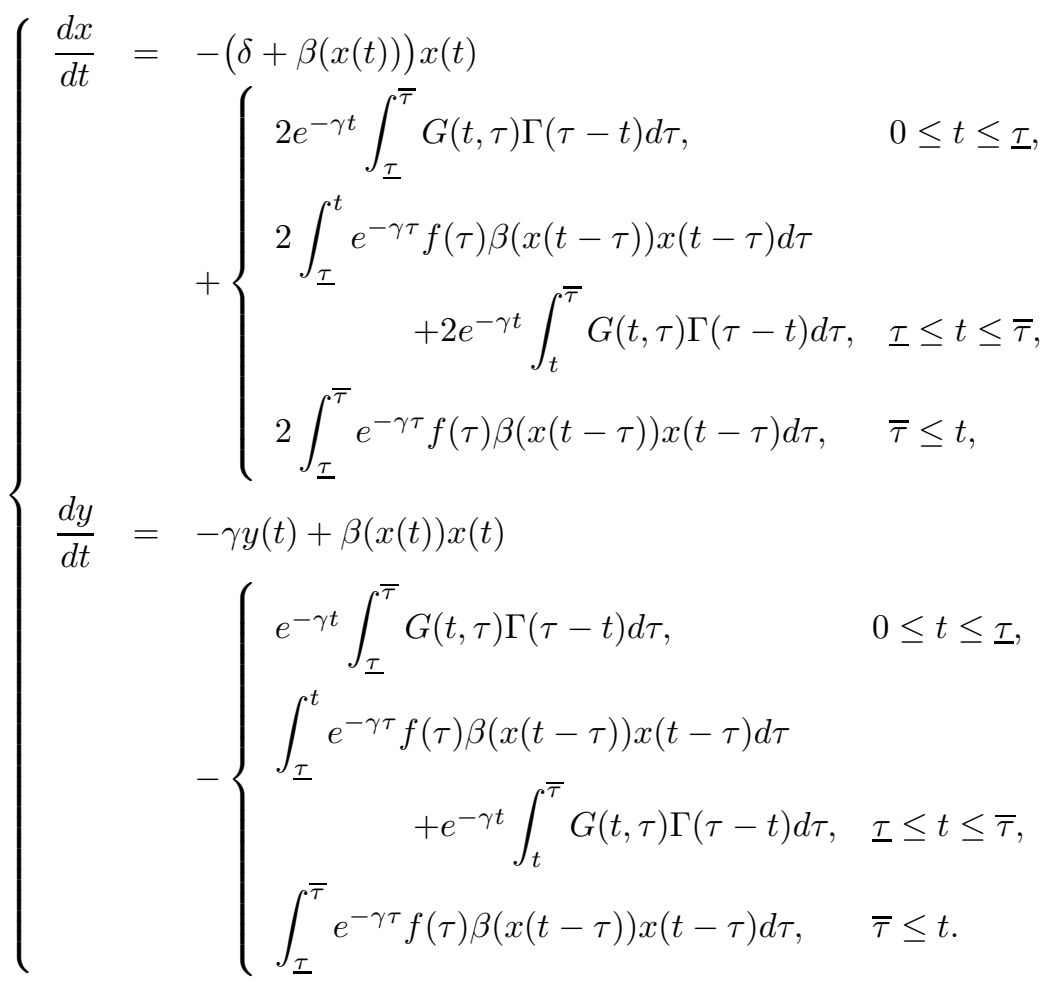

One can give a direct biological explanation of system (7).

In the equation for the resting cells $x(t)$, the first term in the right-hand side accounts for $G_{0}$-cell loss due to either the mortality and cellular differentiation $(\delta)$ or introduction in the proliferating phase $(\beta)$. The second term represents a cellular gain due to the movement of proliferating cells one generation earlier. It requires some explanations. First, we recall that all cells divide according to the density $f$, supported on $[\underline{\tau}, \bar{\tau}]$. We shall call, in the following, new proliferating cells, the resting cells introduced in the proliferating phase at the considered time $t$. When $t \leq \tau$, no new proliferating cell is mature enough to divide, because cells cannot divide before they have spent a time $\underline{\tau}$ in the proliferating phase. Therefore, the cellular gain can only proceed from cells initially in the proliferating phase. When $t \in[\underline{\tau}, \bar{\tau}]$, the cellular increase is obtained by division of new proliferating cells and by division of the initial population. Finally, when $t \geq \bar{\tau}$, all initial proliferating cells have divided or died, and the cellular gain is obtained by division of new proliferating cells introduced one generation earlier. The factor 2 always accounts for the division of each cell into two daughter cells at mitosis. The term $e^{-\gamma t}$, with $t \in[0, \bar{\tau}]$, describes the attenuation of the population, in the proliferating phase, due to apoptosis.

In the equation for the proliferating cells $y(t)$, the first term in the right-hand side accounts for cellular loss by apoptosis and the second term is for cellular entry from the $G_{0}$-phase. The last term accounts for the flux of proliferating cells to the resting compartment.

We set $\mu:=\int_{0}^{\infty} \nu(a) d a$. Then, initially, the populations in the two phases are given by

$$
x(0)=\mu \quad \text { and } \quad y(0)=\int_{0}^{\bar{\tau}} \Gamma(a) d a .
$$

At this point, one can make a remark. Since resting cells are introduced in the proliferating phase with a rate $\beta$, then $\Gamma(0)$, which represents the population of cells introduced at time 
$t=0$ in the cycle, must satisfy

$$
\Gamma(0)=\beta(\mu) \mu .
$$

Taking into account the inevitable loss of proliferating cells by apoptosis and by division, we suppose that $\Gamma(a)$ is given by

$$
\Gamma(a)= \begin{cases}e^{-\gamma a} \beta(\mu) \mu, & \text { if } a \in[0, \underline{\tau}), \\ e^{-\gamma a} \exp \left(-\int_{\underline{\underline{\tau}}}^{a} g(s) d s\right) \beta(\mu) \mu, & \text { if } a \in[\underline{\tau}, \bar{\tau}) .\end{cases}
$$

This simply describes that $\Gamma$ satisfies (49) (see Webb [35], page 8). With (8) and integrating by parts, the initial conditions of system (7) become

$$
x(0)=\mu, \quad y(0)=\beta(\mu) \mu \int_{\underline{\tau}}^{\bar{\tau}} f(\tau)\left(\frac{1-e^{-\gamma \tau}}{\gamma}\right) d \tau .
$$

When $\gamma=0$, we have

$$
y(0)=\beta(\mu) \mu \int_{\underline{\tau}}^{\bar{\tau}} \tau f(\tau) d \tau .
$$

Assume that the function $x \mapsto x \beta(x)$ is Lipschitz continuous. It is immediate to show by steps that, for all $\mu \geq 0$, the system (7) under condition (9) has a unique nonnegative continuous solution $(x(t), y(t))$ defined on $[0,+\infty)$.

One can notice that problem (7) reduces to a system of two delay differential equations, with initial conditions solutions of a system of ordinary differential equations. On $[0, \underline{\tau}]$, the first equation for $x(t)$ in system (7) reduces to the ordinary differential equation

$$
\left\{\begin{aligned}
\frac{d \widetilde{\varphi}}{d t} & =-(\delta+\beta(\widetilde{\varphi}(t))) \widetilde{\varphi}(t)+2 \beta(\mu) \mu \int_{\underline{\tau}}^{\bar{\tau}} e^{-\gamma \tau} f(\tau) d \tau, \quad 0 \leq t \leq \underline{\tau}, \\
\widetilde{\varphi}(0) & =\mu,
\end{aligned}\right.
$$

and, on $[\underline{\tau}, \bar{\tau}]$, the second equation reduces to the following nonautonomous delay differential equation

$$
\left\{\begin{array}{rlrl}
\frac{d \varphi}{d t}= & -(\delta+\beta(\varphi(t))) \varphi(t)+2 \beta(\mu) \mu \int_{t}^{\bar{\tau}} e^{-\gamma \tau} f(\tau) d \tau & \\
& +2 \int_{\underline{\tau}}^{t} e^{-\gamma \tau} f(\tau) \beta(\varphi(t-\tau)) \varphi(t-\tau) d \tau, & & t \in[\underline{\tau}, \bar{\tau}], \\
\varphi(t)= & \widetilde{\varphi}(t), & t \in[0, \underline{\tau}],
\end{array}\right.
$$

where $\widetilde{\varphi}(t)$ is the unique solution of (10) for the initial condition $\mu$.

By the same way, the solution $y(t)$ of the second equation in (7), denoted by $\psi(t)$, is given in terms of the unique solution $\widetilde{\varphi}(t)$ of $(10)$, associated with $\mu$, and the unique solution $\varphi(t)$ of (11), for $t \in[0, \bar{\tau}]$.

Then, the system (17) can be written as an autonomous system of delay differential equations, for $t \geq \bar{\tau}$,

$$
\begin{aligned}
& \frac{d x}{d t}=-(\delta+\beta(x(t))) x(t)+2 \int_{\underline{\tau}}^{\bar{\tau}} e^{-\gamma \tau} f(\tau) \beta(x(t-\tau)) x(t-\tau) d \tau, \\
& \frac{d y}{d t}=-\gamma y(t)+\beta(x(t)) x(t)-\int_{\underline{\tau}}^{\bar{\tau}} e^{-\gamma \tau} f(\tau) \beta(x(t-\tau)) x(t-\tau) d \tau,
\end{aligned}
$$


with, for $t \in[0, \bar{\tau}]$,

$$
x(t)=\varphi(t), \quad y(t)=\psi(t) .
$$

The solutions of $(12 \mathrm{~b})$ are given explicitly by

$$
y(t)=\int_{\underline{\tau}}^{\bar{\tau}} f(\tau)\left(\int_{t-\tau}^{t} e^{-\gamma(t-s)} \beta(x(s)) x(s) d s\right) d \tau \quad \text { for } t \geq \bar{\tau} .
$$

One can notice that $y(t)$ does not depend anymore on the initial population $\Gamma(a)$ after one generation, that is when $t \geq \bar{\tau}$. This can be explained as follows: Cells initially in the proliferating phase have divided or died after one generation; hence, new cells in the proliferating phase can only come from resting cells $x(t)$.

On the other hand, one may have already noticed that the solutions of (12a) do not depend on the solutions of $(12 \mathrm{~b})$ whereas the converse is not true. The expression of $y(t)$ in (14) gives more precise information on the influence of the behavior of $x(t)$ on the stability of the solutions $y(t)$. These results are proved in the following lemma.

Lemma 2.1. Let $(x(t), y(t))$ be a solution of (19). If $\lim _{t \rightarrow+\infty} x(t)$ exists and equals $C \geq 0$, then

$$
\lim _{t \rightarrow+\infty} y(t)= \begin{cases}\beta(C) C \int_{\underline{\tau}}^{\bar{\tau}} f(\tau)\left(\frac{1-e^{-\gamma \tau}}{\gamma}\right) d \tau, & \text { if } \gamma>0 \\ \beta(C) C \int_{\underline{\tau}}^{\bar{\tau}} \tau f(\tau) d \tau, & \text { if } \gamma=0 .\end{cases}
$$

If $x(t)$ is $P$-periodic, then $y(t)$ is also P-periodic.

Proof. By using (14), we obtain that

$$
y(t)=\int_{\underline{\tau}}^{\bar{\tau}} f(\tau)\left(\int_{0}^{\tau} e^{-\gamma s} \beta(x(t-s)) x(t-s) d s\right) d \tau \quad \text { for } t \geq \bar{\tau} .
$$

Hence,

$$
\lim _{t \rightarrow+\infty} y(t)=\beta(C) C \int_{\underline{\tau}}^{\bar{\tau}} f(\tau)\left(\int_{0}^{\tau} e^{-\gamma s} d s\right) d \tau
$$

and (15) follows immediately.

When $x(t)$ is $P$-periodic, then using $(16)$ it is obvious to see that $y(t)$ is also periodic with the same period.

Lemma 2.1 shows the influence of (12a) on the stability of the entire system, since the stability of solutions of (12a) leads to stability of the solutions of $(12 \mathrm{~b})$.

Before studying the stability of $12 \mathrm{a}$, we prove a boundedness result for the solutions of this equation. The proof is based on the one given by Mackey and Rudnicki [22] for a differential equation with a discrete delay.

Proposition 2.1. Assume that $\delta>0$. Then the solutions of (12a) are bounded.

Proof. Assume that $\delta>0$ and $2\left(\int_{\underline{\tau}}^{\bar{\tau}} e^{-\gamma \tau} f(\tau) d \tau\right) \beta(0) \geq \delta$. Since $\beta$ is decreasing and $\lim _{x \rightarrow+\infty} \beta(x)=0$, there exists a unique $x_{0} \geq 0$ such that

$$
2\left(\int_{\underline{\tau}}^{\bar{\tau}} e^{-\gamma \tau} f(\tau) d \tau\right) \beta\left(x_{0}\right)=\delta
$$


and

$$
2\left(\int_{\underline{\underline{\tau}}}^{\bar{\tau}} e^{-\gamma \tau} f(\tau) d \tau\right) \beta(x) \leq \delta \quad \text { for } x \geq x_{0} .
$$

If $2\left(\int_{\underline{\underline{\tau}}}^{\bar{\tau}} e^{-\gamma \tau} f(\tau) d \tau\right) \beta(0)<\delta$, then (17) holds with $x_{0}=0$. Set

$$
x_{1}:=2\left(\int_{\underline{\underline{\tau}}}^{\bar{\tau}} e^{-\gamma \tau} f(\tau) d \tau\right) \frac{\beta(0) x_{0}}{\delta} \geq 0 .
$$

One can check that

$$
2\left(\int_{\underline{\underline{\tau}}}^{\bar{\tau}} e^{-\gamma \tau} f(\tau) d \tau\right) \max _{0 \leq y \leq x}(\beta(y) y) \leq \delta x \quad \text { for } x \geq x_{1} .
$$

Indeed, let $y \in[0, x)$. If $y \leq x_{0}$, then

$$
2\left(\int_{\underline{\underline{\tau}}}^{\bar{\tau}} e^{-\gamma \tau} f(\tau) d \tau\right) \beta(y) y \leq 2\left(\int_{\underline{\underline{\tau}}}^{\bar{\tau}} e^{-\gamma \tau} f(\tau) d \tau\right) \beta(0) x_{0}=\delta x_{1} \leq \delta x
$$

and, if $y>x_{0}$, then

$$
2\left(\int_{\underline{\tau}}^{\bar{\tau}} e^{-\gamma \tau} f(\tau) d \tau\right) \beta(y) y \leq \delta y \leq \delta x
$$

Hence, (18) holds.

Assume, by contradiction, that $\lim \sup _{t \rightarrow+\infty} x(t)=+\infty$, where $x(t)$ is a solution of (12a). Then, there exists $t_{0}>\bar{\tau}$ such that

$$
x(t) \leq x\left(t_{0}\right) \text { for } t \in\left[t_{0}-\bar{\tau}, t_{0}\right] \quad \text { and } \quad x\left(t_{0}\right)>x_{1} .
$$

With (18), we obtain that

$$
2 \int_{\underline{\tau}}^{\bar{\tau}} e^{-\gamma \tau} f(\tau) \beta\left(x\left(t_{0}-\tau\right)\right) x\left(t_{0}-\tau\right) d \tau \leq \delta x\left(t_{0}\right) .
$$

This yields, with (12a), that

$$
\left.\frac{d x}{d t}\left(t_{0}\right) \leq-\beta\left(x\left(t_{0}\right)\right)\right) x\left(t_{0}\right)<0,
$$

which gives a contradiction. Hence, $\lim \sup _{t \rightarrow+\infty} x(t)<+\infty$.

When $\delta=0$, the solutions of (12a) may not be bounded. We show, in the next proposition, that these solutions may explode under some conditions. However, one can notice, using (16), that the solutions of (12b) may still be stable in this case.

Proposition 2.2. Assume that $\delta=0$ and

$$
\int_{\underline{\tau}}^{\bar{\tau}} e^{-\gamma \tau} f(\tau) d \tau>\frac{1}{2} .
$$

In addition, assume that there exists $\bar{x} \geq 0$ such that the function $x \mapsto x \beta(x)$ is decreasing for $x \geq \bar{x}$. If $\mu \geq \bar{x}$, then the unique solution $x(t)$ of (12d) satisfies

$$
\lim _{t \rightarrow+\infty} x(t)=+\infty .
$$


Proof. One can notice that, if $\lim _{t \rightarrow+\infty} x(t)=C$ exists, then (12a) leads to

$$
\left(2 \int_{\underline{\tau}}^{\bar{\tau}} e^{-\gamma \tau} f(\tau) d \tau-1\right) \beta(C) C=0 .
$$

It follows that $C=0$.

Let $\mu \geq \bar{x}$ be given. Consider the equation

$$
\widetilde{\varphi}^{\prime}(t)=2 \beta(\mu) \mu \int_{\underline{\tau}}^{\bar{\tau}} e^{-\gamma \tau} f(\tau) d \tau-\beta(\widetilde{\varphi}(t)) \widetilde{\varphi}(t) \quad \text { for } 0 \leq t \leq \underline{\tau}
$$

with $\widetilde{\varphi}(0)=\mu$. Since the function $x \mapsto x \beta(x)$ is decreasing for $x \geq \bar{x}$, it is immediate that every solution $\widetilde{\varphi}(t)$ of (20) satisfies, for $t \in[0, \underline{\tau}]$,

$$
\widetilde{\varphi}^{\prime}(t) \geq\left(2 \int_{\underline{\tau}}^{\bar{\tau}} e^{-\gamma \tau} f(\tau) d \tau-1\right) \beta(\mu) \mu>0 .
$$

Consider now the problem

$$
\left\{\begin{array}{rlrl}
\varphi^{\prime}(t)= & -\beta(\varphi(t)) \varphi(t)+2 \beta(\mu) \mu \int_{t}^{\bar{\tau}} e^{-\gamma \tau} f(\tau) d \tau & \\
& +2 \int_{\underline{\tau}}^{t} e^{-\gamma \tau} f(\tau) \beta(\varphi(t-\tau)) \varphi(t-\tau) d \tau, & & t \in[\underline{\tau}, \bar{\tau}] \\
\varphi(t)= & \widetilde{\varphi}(t), & & t \in[0, \underline{\tau}]
\end{array}\right.
$$

where $\widetilde{\varphi}(t)$ is the unique solution of (20) for the initial condition $\mu$. Then,

$$
\varphi^{\prime}(\underline{\tau}) \geq\left(2 \int_{\underline{\tau}}^{\bar{\tau}} e^{-\gamma \tau} f(\tau) d \tau-1\right) \beta(\mu) \mu>0 .
$$

So, there exists $\varepsilon>0$ such that $\underline{\tau}+\varepsilon \leq \bar{\tau}$ and $\varphi^{\prime}(t)>0$ for $t \in[\underline{\tau}, \underline{\tau}+\varepsilon)$. Since $\mu \leq \varphi(\underline{\tau}) \leq$ $\varphi(\tau) \leq \varphi(\underline{\tau}+\varepsilon)$, for $\tau \in[\underline{\tau}, \underline{\tau}+\varepsilon]$, we have

$$
\begin{aligned}
\varphi^{\prime}(\underline{\tau}+\varepsilon) \geq & \left(2 \int_{\underline{\tau}+\varepsilon}^{\bar{\tau}} e^{-\gamma \tau} f(\tau) d \tau-1\right) \beta(\varphi(\underline{\tau}+\varepsilon)) \varphi(\underline{\tau}+\varepsilon) \\
& +2\left(\int_{\underline{\tau}}^{\underline{\tau}+\varepsilon} e^{-\gamma \tau} f(\tau) d \tau\right) \beta(\varphi(\underline{\tau}+\varepsilon)) \varphi(\underline{\tau}+\varepsilon) \\
\geq & \left(2 \int_{\underline{\tau}}^{\bar{\tau}} e^{-\gamma \tau} f(\tau) d \tau-1\right) \beta(\varphi(\underline{\tau}+\varepsilon)) \varphi(\underline{\tau}+\varepsilon) .
\end{aligned}
$$

Condition (19) leads to $\varphi^{\prime}(\underline{\tau}+\varepsilon)>0$. Using a similar argument, we obtain that

$$
\varphi^{\prime}(t)>0 \quad \text { for } t \in[\underline{\tau}, \bar{\tau}] .
$$

To conclude, consider the delay differential equation

$$
x^{\prime}(t)=2 \int_{\underline{\tau}}^{\bar{\tau}} e^{-\gamma \tau} f(\tau) \beta(x(t-\tau)) x(t-\tau) d \tau-\beta(x(t)) x(t)
$$

with an initial condition given on $[\underline{\tau}, \bar{\tau}]$ by the solution $\varphi(t)$ of $(21)$. Using the same reasoning as in the previous cases, we obtain that

$$
x^{\prime}(\bar{\tau})>0 .
$$


We thus deduce that

$$
x^{\prime}(t)>0 \quad \text { for } t \geq 0 .
$$

This completes the proof.

The assumption on the function $x \mapsto x \beta(x)$ in Proposition 2.2 is satisfied for example when $\beta$ is given by (1D), with $n>1$. In this case, we can take $\bar{x}=\theta /(n-1)^{1 / n}$.

We now turn our attention to the stability of (12). Problem (12) has at most two equilibria. The first one, $E_{0}=(0,0)$, always exists: it corresponds to the extinction of the population. The second one describes the expected equilibrium of the population; it is a nontrivial equilibrium $E^{*}=\left(x^{*}, y^{*}\right)$, where $x^{*}$ is the unique solution of

$$
\left(2 \int_{\underline{\tau}}^{\bar{\tau}} e^{-\gamma \tau} f(\tau) d \tau-1\right) \beta\left(x^{*}\right)=\delta
$$

and, from (7) and (9),

$$
y^{*}= \begin{cases}\beta\left(x^{*}\right) x^{*} \int_{\underline{\tau}}^{\bar{\tau}} f(\tau)\left(\frac{1-e^{-\gamma \tau}}{\gamma}\right) d \tau, & \text { if } \gamma>0, \\ \delta x^{*} \int_{\underline{\tau}}^{\bar{\tau}} \tau f(\tau) d \tau, & \text { if } \gamma=0 .\end{cases}
$$

Since $\beta$ is a positive decreasing function and $\lim _{x \rightarrow+\infty} \beta(x)=0$, then the equilibrium $E^{*}$ exists if and only if

$$
0<\delta<\left(2 \int_{\underline{\tau}}^{\bar{\tau}} e^{-\gamma \tau} f(\tau) d \tau-1\right) \beta(0) .
$$

We shall study in Section 3 the stability of the two equilibria $E_{0}$ and $E^{*}$. From Lemma 2.1, we only need to focus on the behavior of the equilibria of (12a), that is $x \equiv 0$ and $x \equiv x^{*}$, to obtain information on the behavior of the entire population.

\section{$3 \quad$ Asymptotic stability}

We first show that $E_{0}$ is globally asymptotically stable when it is the only equilibrium, and that it becomes unstable when the nontrivial equilibrium $E^{*}$ appears: a transcritical bifurcation occurs then. In a second part, we determine conditions for the nontrivial equilibrium $E^{*}$ to be asymptotically stable.

\subsection{Stability of the trivial equilibrium}

In the next theorem, we give a necessary and sufficient condition for the trivial equilibrium of (12a) to be globally asymptotically stable using a Lyapunov functional. Concerning definition and interest of Lyapunov functionals for delay differential equations, we refer to Hale [13].

Theorem 3.1. The trivial equilibrium of the system (19) is globally asymptotically stable if

$$
\left(2 \int_{\underline{\tau}}^{\bar{\tau}} e^{-\gamma \tau} f(\tau) d \tau-1\right) \beta(0)<\delta
$$

and unstable if

$$
\delta<\left(2 \int_{\underline{\underline{\tau}}}^{\bar{\tau}} e^{-\gamma \tau} f(\tau) d \tau-1\right) \beta(0) .
$$


Proof. We first assume that (26) holds. Denote by $C^{+}$the set of continuous nonnegative functions on $[0, \bar{\tau}]$ and define the mapping $J: C^{+} \rightarrow[0,+\infty)$ by

$$
J(\varphi)=B(\varphi(\bar{\tau}))+\int_{\underline{\tau}}^{\bar{\tau}} e^{-\gamma \tau} f(\tau)\left(\int_{\bar{\tau}-\tau}^{\bar{\tau}}(\beta(\varphi(\theta)) \varphi(\theta))^{2} d \theta\right) d \tau
$$

for all $\varphi \in C^{+}$, where

$$
B(x)=\int_{0}^{x} \beta(s) s d s \quad \text { for all } x \geq 0 .
$$

We set (see [13])

$$
\dot{J}(\varphi)=\limsup _{t \rightarrow 0^{+}} \frac{J\left(x_{t}^{\varphi}\right)-J(\varphi)}{t} \quad \text { for } \varphi \in C^{+},
$$

where $x^{\varphi}$ is the unique solution of (12a) associated with the initial condition $\varphi \in C^{+}$, and $x_{t}^{\varphi}(\theta)=x^{\varphi}(t+\theta)$ for $\theta \in[0, \bar{\tau}]$. Then,

$$
\begin{aligned}
\dot{J}(\varphi)= & \frac{d \varphi}{d t}(\bar{\tau}) \beta(\varphi(\bar{\tau})) \varphi(\bar{\tau}) \\
& +\int_{\underline{\tau}}^{\bar{\tau}} e^{-\gamma \tau} f(\tau)\left((\beta(\varphi(\bar{\tau})) \varphi(\bar{\tau}))^{2}-(\beta(\varphi(\bar{\tau}-\tau)) \varphi(\bar{\tau}-\tau))^{2}\right) d \tau
\end{aligned}
$$

Using (12a), we have

$$
\frac{d \varphi}{d t}(\bar{\tau})=-(\delta+\beta(\varphi(\bar{\tau}))) \varphi(\bar{\tau})+2 \int_{\underline{\tau}}^{\bar{\tau}} e^{-\gamma \tau} f(\tau) \beta(\varphi(\bar{\tau}-\tau)) \varphi(\bar{\tau}-\tau) d \tau
$$

Therefore, (28) becomes

$$
\begin{aligned}
\dot{J}(\varphi)= & -(\delta+\beta(\varphi(\bar{\tau}))) \beta(\varphi(\bar{\tau})) \varphi^{2}(\bar{\tau})+\int_{\underline{\tau}}^{\bar{\tau}} e^{-\gamma \tau} f(\tau)\left[(\beta(\varphi(\bar{\tau})) \varphi(\bar{\tau}))^{2}\right. \\
& \left.+2 \beta(\varphi(\bar{\tau})) \varphi(\bar{\tau}) \beta(\varphi(\bar{\tau}-\tau)) \varphi(\bar{\tau}-\tau)-(\beta(\varphi(\bar{\tau}-\tau)) \varphi(\bar{\tau}-\tau))^{2}\right] d \tau \\
= & -(\delta+\beta(\varphi(\bar{\tau}))) \beta(\varphi(\bar{\tau})) \varphi^{2}(\bar{\tau})+2(\beta(\varphi(\bar{\tau})) \varphi(\bar{\tau}))^{2} \int_{\underline{\tau}}^{\bar{\tau}} e^{-\gamma \tau} f(\tau) d \tau \\
& -\int_{\underline{\tau}}^{\bar{\tau}} e^{-\gamma \tau} f(\tau)[\beta(\varphi(\bar{\tau})) \varphi(\bar{\tau})-\beta(\varphi(\bar{\tau}-\tau)) \varphi(\bar{\tau}-\tau)]^{2} d \tau .
\end{aligned}
$$

Hence,

$$
\dot{J}(\varphi) \leq-u(\varphi(\bar{\tau}))
$$

where the function $u$ is defined, for $x \geq 0$, by

$$
u(x)=r(x) \beta(x) x^{2}
$$

with

$$
r(x)=\delta-\left(2 \int_{\underline{\tau}}^{\bar{\tau}} e^{-\gamma \tau} f(\tau) d \tau-1\right) \beta(x) .
$$

Since $\beta$ is decreasing, $r$ is a monotone function. Moreover, (26) leads to $r(0)>0$, and $\lim _{x \rightarrow \infty} r(x)=\delta \geq 0$. Therefore, $r$ is positive on $[0,+\infty)$. 
Consequently, the function $u$ defined by (29) is nonnegative on $[0,+\infty)$ and $u(x)=0$ if and only if $x=0$. We deduce that every solution of (12a), with $\varphi \in C^{+}$, tends to zero as $t$ tends to $+\infty$.

We suppose now that (27) holds. The linearization of (12a) around $x \equiv 0$ leads to the characteristic equation

$$
\Delta_{0}(\lambda):=\lambda+\delta+\beta(0)-2 \beta(0) \int_{\underline{\tau}}^{\bar{\tau}} e^{-(\lambda+\gamma) \tau} f(\tau) d \tau=0 .
$$

We consider $\Delta_{0}$ as a real function. Since

$$
\frac{d \Delta_{0}}{d \lambda}=1+2 \beta(0) \int_{\underline{\tau}}^{\bar{\tau}} \tau e^{-(\lambda+\gamma) \tau} f(\tau) d \tau>0
$$

it follows that $\Delta_{0}$ is an increasing function. Moreover, (30) yields

$$
\lim _{\lambda \rightarrow-\infty} \Delta_{0}(\lambda)=-\infty, \quad \lim _{\lambda \rightarrow+\infty} \Delta_{0}(\lambda)=+\infty,
$$

and (27) implies that

$$
\Delta_{0}(0)=\delta-\left(2 \int_{\underline{\tau}}^{\bar{\tau}} e^{-\gamma \tau} f(\tau) d \tau-1\right) \beta(0)<0
$$

Hence, $\Delta_{0}(\lambda)$ has a unique real root which is positive. Consequently, (30) has at least one characteristic root with positive real part. Therefore, the equilibrium $x \equiv 0$ of (12a) is not stable. This completes the proof.

The inequality (26) is satisfied when $\delta$ or $\gamma$ (the mortality rates) is large or when $\beta(0)$ is small. Biologically, these conditions correspond to a population which cannot survive, because the mortality rates are too large or, simply, because not enough cells are introduced in the proliferating phase and, then, the population renewal is not supplied.

Remark 1. One can notice that when

$$
\int_{\underline{\tau}}^{\bar{\tau}} e^{-\gamma \tau} f(\tau) d \tau<\frac{1}{2}
$$

the trivial equilibrium $E_{0}$ is the only equilibrium of 12 and is globally asymptotically stable. When

$$
\int_{\underline{\tau}}^{\bar{\tau}} e^{-\gamma \tau} f(\tau) d \tau=\frac{1}{2}
$$

then $E_{0}$ is globally asymptotically stable if $\delta>0$. When the equality

$$
\left(2 \int_{\underline{\tau}}^{\bar{\tau}} e^{-\gamma \tau} f(\tau) d \tau-1\right) \beta(0)=\delta
$$

holds, one can check that $\lambda=0$ is a characteristic root of (30) and all other characteristic roots have negative real parts. Hence, we cannot conclude on the stability or instability of the trivial equilibrium $E_{0}$ of (12) without further analysis. However, this is not the subject of this paper. 


\subsection{Stability of the nontrivial equilibrium}

We concentrate, in this section, on the equilibrium $E^{*}=\left(x^{*}, y^{*}\right)$ defined by (23)-(24). Hence, throughout this section, we assume that (25) holds, that is

$$
0<\delta<\left(2 \int_{\underline{\tau}}^{\bar{\tau}} e^{-\gamma \tau} f(\tau) d \tau-1\right) \beta(0)
$$

Since $\delta>0$ and $\beta(0)>0,25)$ implies, in particularly, that

$$
\int_{\underline{\tau}}^{\bar{\tau}} e^{-\gamma \tau} f(\tau) d \tau>\frac{1}{2}
$$

From Lemma 2.1, we only need to focus on the stability of the nontrivial equilibrium $x \equiv x^{*}$ of (12a). To that aim, we linearize (12a) around $x^{*}$. Denote by $\beta^{*} \in \mathbb{R}$ the quantity

$$
\beta^{*}:=\left.\frac{d}{d x}(x \beta(x))\right|_{x=x^{*}}=\beta\left(x^{*}\right)+x^{*} \beta^{\prime}\left(x^{*}\right)
$$

and set $u(t)=x(t)-x^{*}$. The linearization of $(12 \mathrm{a})$ is given by

$$
\frac{d u}{d t}=-\left(\delta+\beta^{*}\right) u(t)+2 \beta^{*} \int_{\underline{\tau}}^{\bar{\tau}} e^{-\gamma \tau} f(\tau) u(t-\tau) d \tau .
$$

Then, the characteristic equation is

$$
\Delta(\lambda):=\lambda+\delta+\beta^{*}-2 \beta^{*} \int_{\underline{\tau}}^{\bar{\tau}} e^{-(\lambda+\gamma) \tau} f(\tau) d \tau=0
$$

One can notice that the function $x \mapsto x \beta(x)$ is usually not monotone. For example, if $\beta$ is given by (11) with $n>1$, the function $x \mapsto x \beta(x)$ is increasing for $x \leq \theta /(n-1)^{1 / n}$ and decreasing for $x>\theta /(n-1)^{1 / n}$. In this case, $\beta^{*}$ is nonnegative when $x^{*}$ is close to zero and negative when $x^{*}$ is large enough.

The following theorem deals with the asymptotic stability of $E^{*}$.

Theorem 3.2. Assume that (25) holds. If

$$
\beta^{*} \geq-\frac{\delta}{2 \int_{\underline{\tau}}^{\bar{\tau}} e^{-\gamma \tau} f(\tau) d \tau+1},
$$

then $E^{*}$ is locally asymptotically stable.

Proof. We first prove that the equilibrium $x \equiv x^{*}$ is locally asymptotically stable when $\beta^{*} \geq 0$. We consider the mapping $\Delta(\lambda)$, given by (33), as a real function of $\lambda$. Then $\Delta(\lambda)$ is continuously differentiable on $\mathbb{R}$ and its first derivative is given by

$$
\frac{d \Delta}{d \lambda}=1+2 \beta^{*} \int_{\underline{\tau}}^{\bar{\tau}} \tau e^{-(\lambda+\gamma) \tau} f(\tau) d \tau>0 .
$$

Hence, $\Delta(\lambda)$ is an increasing function of $\lambda$ satisfying

$$
\lim _{\lambda \rightarrow-\infty} \Delta(\lambda)=-\infty \quad \text { and } \quad \lim _{\lambda \rightarrow+\infty} \Delta(\lambda)=+\infty
$$


Then, there exists a unique $\lambda_{0} \in \mathbb{R}$ such that $\Delta\left(\lambda_{0}\right)=0$. Moreover, since

$$
\Delta(0)=\delta-\left(2 \int_{\underline{\tau}}^{\bar{\tau}} e^{-\gamma \tau} f(\tau) d \tau-1\right) \beta^{*},
$$

we deduce, by using (23), (31) and (32), that

$$
\Delta(0)=-\left(2 \int_{\underline{\tau}}^{\bar{\tau}} e^{-\gamma \tau} f(\tau) d \tau-1\right) x^{*} \beta^{\prime}\left(x^{*}\right)>0 .
$$

Consequently, $\lambda_{0}<0$.

Let $\lambda=\mu+i \omega$ be a characteristic root of (33) such that $\mu>\lambda_{0}$. Considering the real part of (33), we obtain that

$$
\mu=-\left(\delta+\beta^{*}\right)+2 \beta^{*} \int_{\underline{\tau}}^{\bar{\tau}} e^{-(\mu+\gamma) \tau} f(\tau) \cos (\omega \tau) d \tau .
$$

Using (33), with $\lambda=\lambda_{0}$, together with (36), we then obtain

$$
\mu-\lambda_{0}=2 \beta^{*} \int_{\underline{\tau}}^{\bar{\tau}} e^{-\gamma \tau} f(\tau)\left[e^{-\mu \tau} \cos (\omega \tau)-e^{-\lambda_{0} \tau}\right] d \tau
$$

However,

$$
e^{-\mu \tau} \cos (\omega \tau)-e^{-\lambda_{0} \tau}<0
$$

for all $\tau \in[\underline{\tau}, \bar{\tau}]$. So we obtain that $\mu-\lambda_{0}<0$, which leads to a contradiction. This implies that all characteristic roots of (33) have negative real part and the equilibrium $x \equiv x^{*}$ of (12a) is locally asymptotically stable.

Now, assume that $\beta^{*}<0$ and

$$
\beta^{*}>-\frac{\delta}{2 \int_{\underline{\tau}}^{\bar{\tau}} e^{-\gamma \tau} f(\tau) d \tau+1} .
$$

Let $\lambda=\mu+i \omega$ be a characteristic root of (33) such that $\mu>0$. Since

$$
\int_{\underline{\tau}}^{\bar{\tau}} e^{-\gamma \tau} f(\tau)\left(e^{-\mu \tau} \cos (\omega \tau)+1\right) d \tau \geq 0
$$

we have

$$
2 \beta^{*} \int_{\underline{\tau}}^{\bar{\tau}} e^{-(\mu+\gamma) \tau} f(\tau) \cos (\omega \tau) d \tau \leq-2 \beta^{*} \int_{\underline{\tau}}^{\bar{\tau}} e^{-\gamma \tau} f(\tau) d \tau
$$

So, (36) and (37) lead to

$$
\mu \leq-\left(\delta+\beta^{*}\right)-2 \beta^{*} \int_{\underline{\tau}}^{\bar{\tau}} e^{-\gamma \tau} f(\tau) d \tau<0,
$$

a contradiction. Therefore, $\mu \leq 0$.

Suppose now that (33) has a purely imaginary characteristic root $i \omega$, with $\omega \in \mathbb{R}$. Then, (36) leads to

$$
\int_{\underline{\tau}}^{\bar{\tau}} e^{-\gamma \tau} f(\tau) \cos (\omega \tau) d \tau=\frac{\delta+\beta^{*}}{2 \beta^{*}}
$$


However,

$$
\left|\int_{\underline{\tau}}^{\bar{\tau}} e^{-\gamma \tau} f(\tau) \cos (\omega \tau) d \tau\right| \leq \int_{\underline{\tau}}^{\bar{\tau}} e^{-\gamma \tau} f(\tau) d \tau
$$

and (37) yields

$$
\frac{\delta+\beta^{*}}{2 \beta^{*}}<-\int_{\underline{\tau}}^{\bar{\tau}} e^{-\gamma \tau} f(\tau) d \tau
$$

Hence, (33) has no purely imaginary root. Consequently, all characteristic roots of (33) have negative real part and the nontrivial equilibrium $x \equiv x^{*}$ of $12 \mathrm{a}$ is locally asymptotically stable.

Finally, assume that

$$
\beta^{*}=-\frac{\delta}{2 \int_{\underline{\tau}}^{\bar{\tau}} e^{-\gamma \tau} f(\tau) d \tau+1}
$$

Consider a characteristic root $\lambda=\mu+i \omega$ of (33), which reduces, with (38), to

$$
\lambda-2 \beta^{*} \int_{\underline{\tau}}^{\bar{\tau}} e^{-\gamma \tau} f(\tau)\left(1+e^{-\lambda \tau}\right) d \tau=0 .
$$

Suppose, by contradiction, that $\mu>0$. By considering the real part of (39), we have

$$
\mu=2 \beta^{*} \int_{\underline{\tau}}^{\bar{\tau}} e^{-\gamma \tau} f(\tau)\left(1+e^{-\mu \tau} \cos (\omega \tau)\right) d \tau<0 .
$$

We obtain a contradiction, therefore $\mu \leq 0$. If we suppose now that $\mu=0$ then we easily obtain that

$$
\cos (\omega \tau)=-1 \quad \text { for all } \tau \in[\underline{\tau}, \bar{\tau}]
$$

which is impossible. It follows that all characteristic roots of (33) have negative real parts when (38) holds and the equilibrium $x \equiv x^{*}$ is locally asymptotically stable.

From Lemma 2.1, we conclude that $E^{*}$ is locally asymptotically stable when (34) holds.

The asymptotic stability of $E^{*}$ is shown in Fig. 1. Values of the parameters are given by (2), except $n=2.42, \underline{\tau}=0$ and $\bar{\tau}=7$ days. The function $f$ is defined by

$$
f(\tau)= \begin{cases}\frac{1}{\bar{\tau}-\underline{\tau}}, & \text { if } \tau \in[\underline{\tau}, \bar{\tau}] \\ 0, & \text { otherwise }\end{cases}
$$

The Matlab solver for delay differential equations, dde23 32], is used to obtain Fig. 1, as well as illustrations in Section 1 and Section 5 .

When (34) does not hold, we have necessarily $\beta^{*}<0$. In this case, we cannot obtain the stability of $E^{*}$ for all values of $\beta^{*}$. In fact, in the next section we are going to show that the equilibrium $E^{*}$ can be destabilized, in this case, via a Hopf bifurcation. 


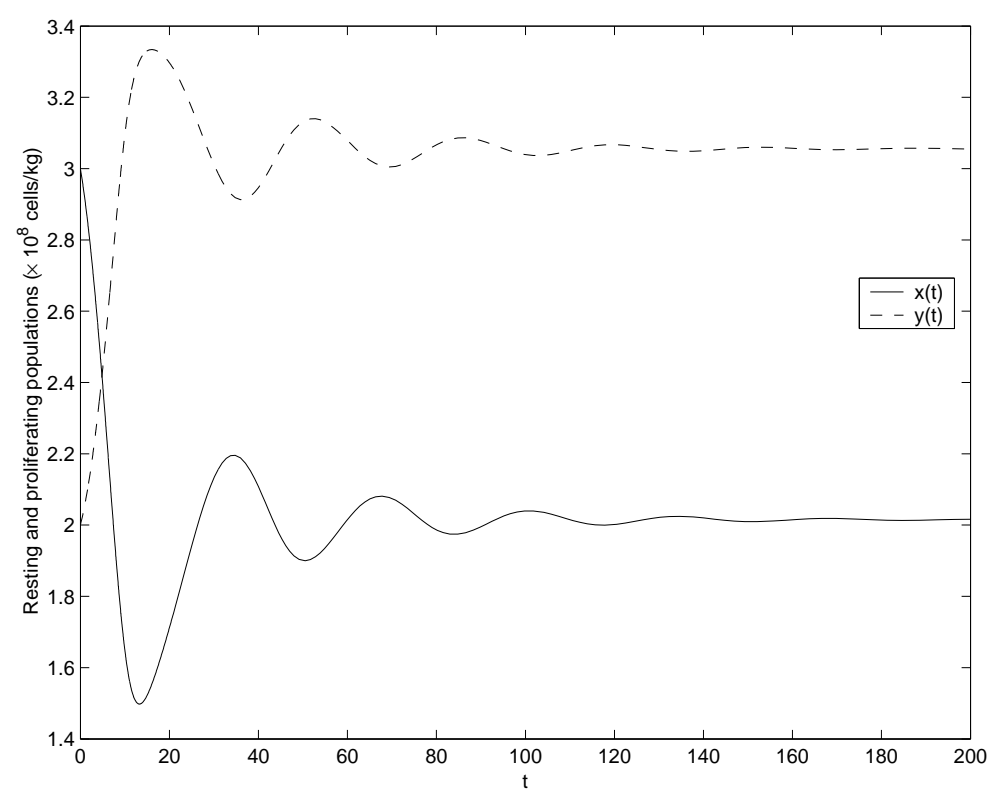

Figure 1: The solutions $x(t)$ (solid curve) and $y(t)$ (dashed curve) of system (12) are drawn for values of the parameters $\beta_{0}, \delta$ and $\gamma$ given by (2), $n=2.42, \underline{\tau}=0$ and $\bar{\tau}=7$ days. In this case, the nontrivial equilibrium $E^{*}$ is locally asymptotically stable, even though the solutions oscillate transiently.

\section{Hopf bifurcation and periodic solutions}

In this section, we show that the equilibrium $x \equiv x^{*}$ of (12a) can become unstable when (34) does not hold anymore. Throughout this section, we assume that

$$
\underline{\tau}=0
$$

and (25) holds, that is

$$
0<\delta<\left(2 \int_{0}^{\bar{\tau}} e^{-\gamma \tau} f(\tau) d \tau-1\right) \beta(0)
$$

From Proposition 2.1, the solutions of (12a) are bounded. Consequently, instability in (12a) occurs only via oscillatory solutions.

We assume that

$$
\beta^{*}<-\frac{\delta}{2 \int_{0}^{\bar{\tau}} e^{-\gamma \tau} f(\tau) d \tau+1}:=\widetilde{\delta} .
$$

Otherwise, the nontrivial equilibrium $x \equiv x^{*}$ of (12a) is locally asymptotically stable (see Theorem 3.2).

If instability occurs for a particular value $\beta^{*}<\widetilde{\delta}$, a characteristic root of (33) must intersect the imaginary axis. Hence, we look for purely imaginary characteristic roots $i \omega$, $\omega \in \mathbb{R}$, of (33). If $i \omega$ is a characteristic root of (33), then $\omega$ is a solution of the system

$$
\left\{\begin{array}{r}
\delta+\beta^{*}(1-2 C(\omega))=0 \\
\omega+2 \beta^{*} S(\omega)=0
\end{array}\right.
$$


where

$$
C(\omega):=\int_{0}^{\bar{\tau}} e^{-\gamma \tau} f(\tau) \cos (\omega \tau) d \tau \quad \text { and } \quad S(\omega):=\int_{0}^{\bar{\tau}} e^{-\gamma \tau} f(\tau) \sin (\omega \tau) d \tau
$$

One can notice that $\omega=0$ is not a solution of (42). Otherwise,

$$
\delta=\left(2 \int_{0}^{\bar{\tau}} e^{-\gamma \tau} f(\tau) d \tau-1\right) \beta^{*}<0
$$

which gives a contradiction. Moreover, if $\omega$ is a solution of (42), then $-i \omega$ is also a characteristic root. Thus, we only look for positive solutions $\omega$.

Lemma 4.1. Assume that the function $\tau \mapsto e^{-\gamma \tau} f(\tau)$ is decreasing. Then, for each $\delta$ such that (25) is satisfied, (42) has at least one solution $\left(\beta_{c}^{*}, \omega_{c}\right)$, with $\beta_{c}^{*}<\widetilde{\delta}$ and $\omega_{c}>0$. It follows that (33) has at least one pair of purely imaginary roots $\pm i \omega_{c}$ for $\beta^{*}=\beta_{c}^{*}$. Moreover, $\pm i \omega_{c}$ are simple characteristic roots of 33 . Consider the branch of characteristic roots $\lambda\left(-\beta^{*}\right)$, such that $\lambda\left(-\beta_{c}^{*}\right)=i \omega_{c}$. Then

$$
\left.\frac{d \operatorname{Re}(\lambda)}{d\left(-\beta^{*}\right)}\right|_{\beta^{*}=\beta_{c}^{*}}>0 \quad \text { if and only if } \quad-\delta\left(\frac{S\left(\omega_{c}\right)}{\omega_{c}}\right)^{\prime}>C^{\prime}\left(\omega_{c}\right) .
$$

Proof. First, we show by induction that $S(\omega)>0$ for $\omega>0$. It is clear that $S(\omega)>0$ if $\omega \bar{\tau} \in(0, \pi]$. Suppose that $\omega \bar{\tau} \in(\pi, 2 \pi]$. Then

$$
\begin{aligned}
S(\omega) & =\frac{1}{\omega} \int_{0}^{\omega \bar{\tau}} e^{-\gamma \frac{\tau}{\omega}} f\left(\frac{\tau}{\omega}\right) \sin (\tau) d \tau \\
& =\frac{1}{\omega} \int_{0}^{\pi} e^{-\gamma \frac{\tau}{\omega}} f\left(\frac{\tau}{\omega}\right) \sin (\tau) d \tau+\frac{1}{\omega} \int_{\pi}^{\omega \bar{\tau}} e^{-\gamma \frac{\tau}{\omega}} f\left(\frac{\tau}{\omega}\right) \sin (\tau) d \tau .
\end{aligned}
$$

Since $f$ is supported on the interval $[0, \bar{\tau}]$, it follows that

$$
\int_{\omega \bar{\tau}}^{2 \pi} e^{-\gamma \frac{\tau}{\omega}} f\left(\frac{\tau}{\omega}\right) \sin (\tau) d \tau=0
$$

So, we obtain

$$
\begin{aligned}
S(\omega) & =\frac{1}{\omega} \int_{0}^{\pi} e^{-\gamma \frac{\tau}{\omega}} f\left(\frac{\tau}{\omega}\right) \sin (\tau) d \tau+\frac{1}{\omega} \int_{\pi}^{2 \pi} e^{-\gamma \frac{\tau}{\omega}} f\left(\frac{\tau}{\omega}\right) \sin (\tau) d \tau \\
& =\frac{1}{\omega} \int_{0}^{\pi}\left(e^{-\gamma \frac{\tau}{\omega}} f\left(\frac{\tau}{\omega}\right)-e^{-\gamma \frac{\tau+\pi}{\omega}} f\left(\frac{\tau+\pi}{\omega}\right)\right) \sin (\tau) d \tau
\end{aligned}
$$

Since the function $\tau \mapsto e^{-\gamma \tau} f(\tau)$ is decreasing, we finally get $S(\omega)>0$. Using a similar argument for $\omega \bar{\tau} \in(k \pi,(k+1) \pi]$, with $k \in \mathbb{N}, k \geq 2$, we deduce that $S(\omega)>0$ for all $\omega>0$.

Consider the equation

$$
g(\omega):=\frac{\omega(1-2 C(\omega))}{2 S(\omega)}=\delta, \quad \omega>0 .
$$

The function $g$ is continuous with

$$
\lim _{\omega \rightarrow 0} g(\omega)=\frac{1-2 C(0)}{2 \int_{0}^{\bar{\tau}} \tau e^{-\gamma \tau} f(\tau) d \tau}<0
$$


because $(25)$ leads to $1-2 C(0)<0$. Moreover, the Riemann-Lebesgue's lemma implies that

$$
\lim _{\omega \rightarrow+\infty} C(\omega)=\lim _{\omega \rightarrow+\infty} S(\omega)=0
$$

This yields

$$
\lim _{\omega \rightarrow+\infty} g(\omega)=+\infty
$$

We conclude that there exists a solution $\omega_{c}>0$ of (44). Since $S\left(\omega_{c}\right)>0$ and $g\left(\omega_{c}\right)=\delta>0$, we obtain $1-2 C\left(\omega_{c}\right)>0$. Set

$$
\beta_{c}^{*}=-\frac{\delta}{1-2 C\left(\omega_{c}\right)}<0
$$

Since $\left|C\left(\omega_{c}\right)\right|<C(0)$, it follows that

$$
\beta_{c}^{*}<-\frac{\delta}{2 C(0)+1}=\widetilde{\delta}
$$

One can check that $\left(\beta_{c}^{*}, \omega_{c}\right)$ is a solution of 42 . It follows that $\pm i \omega_{c}$ are characteristic roots of (33) for $\beta^{*}=\beta_{c}^{*}$.

Define a branch of characteristic roots $\lambda\left(-\beta^{*}\right)$ of $(33)$ such that $\lambda\left(-\beta_{c}^{*}\right)=i \omega_{c}$. We use the parameter $-\beta^{*}$ because $\beta^{*}<\widetilde{\delta}<0$.

Using (33), we obtain

$$
\left[1+2 \beta^{*} \int_{0}^{\bar{\tau}} \tau e^{-(\lambda+\gamma) \tau} f(\tau) d \tau\right] \frac{d \lambda}{d\left(-\beta^{*}\right)}=1-2 \int_{0}^{\bar{\tau}} e^{-(\lambda+\gamma) \tau} f(\tau) d \tau
$$

If we assume, by contradiction, that $i \omega_{c}$ is not a simple root of (33), then (47) leads to

$$
C\left(\omega_{c}\right)=\frac{1}{2} \quad \text { and } \quad S\left(\omega_{c}\right)=0
$$

Since $S\left(\omega_{c}\right)>0$, we obtain a contradiction. Thus, $i \omega_{c}$ is a simple root of (33).

Moreover, using (47), we have

$$
\left(\frac{d \lambda}{d\left(-\beta^{*}\right)}\right)^{-1}=\frac{1+2 \beta^{*} \int_{0}^{\bar{\tau}} \tau e^{-(\lambda+\gamma) \tau} f(\tau) d \tau}{1-2 \int_{0}^{\bar{\tau}} e^{-(\lambda+\gamma) \tau} f(\tau) d \tau}
$$

Since $\lambda$ is a characteristic root of (33), we also have

$$
1-2 \int_{0}^{\bar{\tau}} e^{-(\lambda+\gamma) \tau} f(\tau) d \tau=-\frac{\lambda+\delta}{\beta^{*}}
$$

So, we deduce

$$
\left(\frac{d \lambda}{d\left(-\beta^{*}\right)}\right)^{-1}=-\beta^{*} \frac{1+2 \beta^{*} \int_{0}^{\bar{\tau}} \tau e^{-(\lambda+\gamma) \tau} f(\tau) d \tau}{\lambda+\delta}
$$


Then,

$$
\begin{aligned}
\left.\operatorname{sign}\left\{\frac{d \operatorname{Re}(\lambda)}{d\left(-\beta^{*}\right)}\right\}\right|_{\beta^{*}=\beta_{c}^{*}} & =\left.\operatorname{sign}\left\{\operatorname{Re}\left(\frac{d \lambda}{d\left(-\beta^{*}\right)}\right)^{-1}\right\}\right|_{\beta^{*}=\beta_{c}^{*}} \\
& =\left.\operatorname{sign}\left\{\operatorname{Re}\left(-\beta^{*} \frac{1+2 \beta^{*} \int_{0}^{\bar{\tau}} \tau e^{-(\lambda+\gamma) \tau} f(\tau) d \tau}{\lambda+\delta}\right)\right\}\right|_{\beta^{*}=\beta_{c}^{*}} \\
& =\operatorname{sign}\left\{-\beta_{c}^{*} \frac{\delta\left(1+2 \beta_{c}^{*} S^{\prime}\left(\omega_{c}\right)\right)+2 \beta_{c}^{*} \omega_{c} C^{\prime}\left(\omega_{c}\right)}{\delta^{2}+\omega_{c}^{2}}\right\} \\
& =\operatorname{sign}\left\{\delta\left(1+2 \beta_{c}^{*} S^{\prime}\left(\omega_{c}\right)\right)+2 \beta_{c}^{*} \omega_{c} C^{\prime}\left(\omega_{c}\right)\right\} .
\end{aligned}
$$

From 46 and the fact that $1-2 C\left(\omega_{c}\right)>0$, this leads to

$$
\begin{aligned}
\left.\operatorname{sign}\left\{\frac{d R e(\lambda)}{d\left(-\beta^{*}\right)}\right\}\right|_{\beta^{*}=\beta_{c}^{*}} & =\operatorname{sign}\left\{1-2 C\left(\omega_{c}\right)-2 \delta S^{\prime}\left(\omega_{c}\right)-2 \omega_{c} C^{\prime}\left(\omega_{c}\right)\right\} \\
& =\operatorname{sign}\left\{2 \omega_{c}\left(-C^{\prime}\left(\omega_{c}\right)-\delta\left(\frac{S\left(\omega_{c}\right)}{\omega_{c}}\right)^{\prime}\right)\right\} \\
& =\operatorname{sign}\left\{-C^{\prime}\left(\omega_{c}\right)-\delta\left(\frac{S\left(\omega_{c}\right)}{\omega_{c}}\right)^{\prime}\right\} .
\end{aligned}
$$

This concludes the proof.

Remark 2. Consider the function $g$ defined by (44) and denote by $\alpha$ the quantity

$$
\alpha:=\left(2 \int_{0}^{\bar{\tau}} e^{-\gamma \tau} f(\tau) d \tau-1\right) \beta(0)
$$

Define the sets

$$
\Omega:=\left\{\omega>0 ; 0<g(\omega)<\alpha \text { and } g^{\prime}(\omega)=0\right\} \quad \text { and } \quad \Lambda:=g(\Omega) .
$$

One can notice that $\Lambda$ is finite (or empty). If $\delta \in(0, \alpha) \backslash \Lambda$, then

$$
\left.\frac{d \operatorname{Re}(\lambda)}{d\left(-\beta^{*}\right)}\right|_{\beta^{*}=\beta_{c}^{*}} \neq 0
$$

Indeed, we have

$$
g^{\prime}(\omega)=-\frac{\omega}{S(\omega)}\left(g(\omega)\left(\frac{S(\omega)}{\omega}\right)^{\prime}+C^{\prime}(\omega)\right), \quad \omega>0
$$

Since $\delta \notin \Lambda$, we have $g^{\prime}\left(\omega_{c}\right) \neq 0$. Moreover, $g\left(\omega_{c}\right)=\delta$. Thus

$$
C^{\prime}\left(\omega_{c}\right) \neq-\delta\left(\frac{S\left(\omega_{c}\right)}{\omega_{c}}\right)^{\prime}
$$

We conclude by using 43 ).

Lemma 4.1, together with Remark 2, allows us to state and prove the following theorem. 
Theorem 4.1. Assume that the function $\tau \mapsto e^{-\gamma \tau} f(\tau)$ is decreasing. Then, for each $\delta \notin \Lambda$ satisfying (25), there exists $\beta_{c}^{*}<\widetilde{\delta}$ such that the equilibrium $x \equiv x^{*}$ is locally asymptotically stable when $\beta_{c}^{*}<\beta^{*} \leq \widetilde{\delta}$ and a Hopf bifurcation occurs at $x \equiv x^{*}$ when $\beta^{*}=\beta_{c}^{*}$.

Proof. First, recall that $x \equiv x^{*}$ is locally asymptotically stable when $\beta^{*}=\widetilde{\delta}$ (see Theorem 3.2). We recall that, from the properties of the function $g$, equation (44) has a finite number of solutions (see Lemma 4.1). We set

$$
\beta_{c}^{*}=-\frac{\delta}{1-2 C\left(\omega_{c}^{*}\right)}
$$

where $\omega_{c}^{*}$ is the smaller positive real such that

$$
\left.C\left(\omega_{c}^{*}\right)=\min \{C(\omega) ; \omega \text { is a solution of } 44)\right\} .
$$

Then, $\beta_{c}^{*}$ is the maximum value of $\beta^{*}$ (as defined in Lemma 4.1) which gives a solution of (42). From Lemma 4.1, (33) has no purely imaginary roots while $\beta_{c}^{*}<\beta^{*} \leq \widetilde{\delta}$. Consequently, Rouché's Theorem [10, p.248] leads to the local asymptotic stability of $x \equiv x^{*}$.

When $\beta^{*}=\beta_{c}^{*}$, (33) has a pair of purely imaginary roots $\pm i \omega_{c}, \omega_{c}>0$ (see Lemma 4.1). Moreover, since $\delta \notin \Lambda$, Remark 2 implies that

$$
\left.\frac{d R e(\lambda)}{d\left(-\beta^{*}\right)}\right|_{\beta^{*}=\beta_{c}^{*}} \neq 0 .
$$

Assume, by contradiction, that

$$
\frac{d R e(\lambda)}{d\left(-\beta^{*}\right)}<0
$$

for $\beta^{*}>\beta_{c}^{*}, \beta^{*}$ close to $\beta_{c}^{*}$. Then there exists a characteristic root $\lambda\left(-\beta^{*}\right)$ such that $\operatorname{Re} \lambda\left(-\beta^{*}\right)>0$. This contradicts the fact that $x \equiv x^{*}$ is locally asymptotically stable when $\beta^{*}>\beta_{c}^{*}$. Thus, we obtain

$$
\left.\frac{d R e(\lambda)}{d\left(-\beta^{*}\right)}\right|_{\beta^{*}=\beta_{c}^{*}}>0 .
$$

This implies the existence of a Hopf bifurcation at $x \equiv x^{*}$ for $\beta^{*}=\beta_{c}^{*}$.

With the values of $\delta, \gamma$ and $\beta_{0}$ given by (2), and $\bar{\tau}=7$ days, (12) has periodic solutions for $\beta_{c}^{*}=-0.3881$ with a period about 33 days. This value of $\beta_{c}^{*}$ corresponds to $n=2.53$ (see Fig. 2 and 3). The function $f$ is given by (40).

The bifurcation parameter was chosen to be $\beta^{*}$ in this study, and the values of $\beta^{*}$ depend strongly on the sensitivity $n$ of the function $\beta(x)$, since all other parameters are fixed by $(25)$. In this model, the sensitivity $n$ plays a crucial role in the appearance of periodic solutions. Pujo-Menjouet and Mackey 27] already noticed the influence of this parameter on system (12) when the delay is constant (or equivalently, when $f$ is a Dirac measure). The sensitivity $n$ describes the way the rate of introduction in the proliferating phase reacts to changes in the resting phase population produced by external stimuli: a release of erythropoietin, for example, or the action of some growth factors.

Of course, the influence of other parameters (like mortality rates $\delta$ and $\gamma$, or the minimum and maximum delays $\underline{\tau}$ and $\bar{\tau}$ ) on the appearance of periodic solutions could be studied. However, since periodic hematological diseases — defined and described in Section 5 are supposed to be due to hormonal control destabilization (see 11), then the parameter $n$, among other parameters, seems to be appropriate to identify causes leading to periodic solutions in (12). 


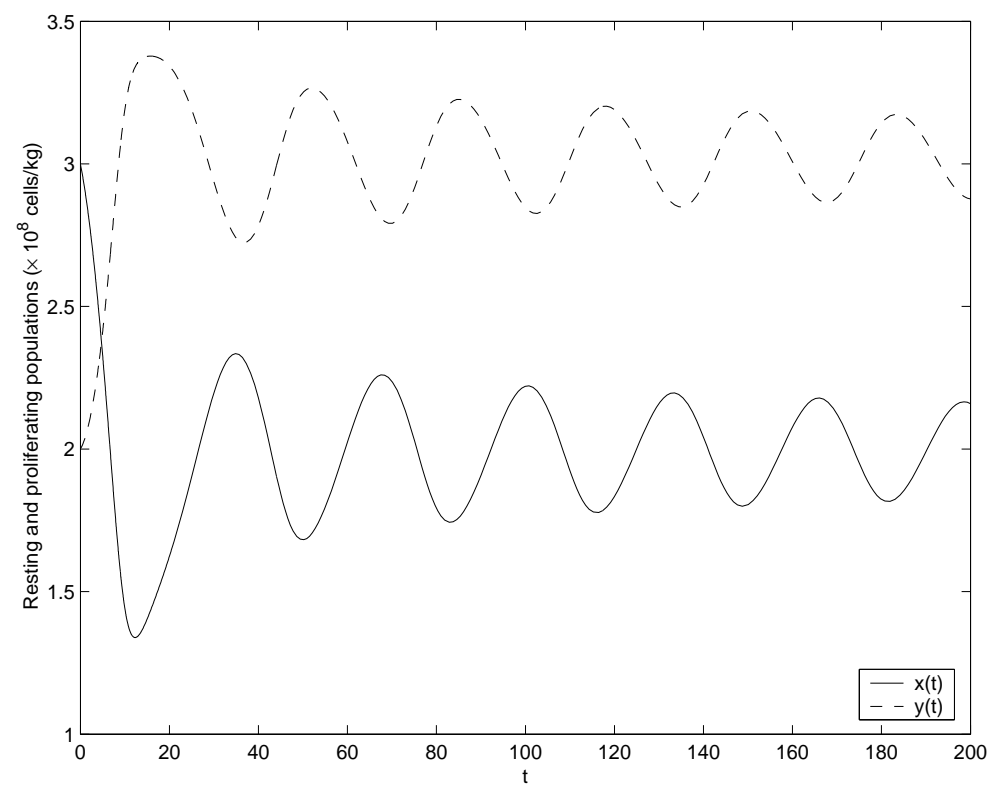

Figure 2: The solutions of system (12), $x(t)$ (solid curve) and $y(t)$ (dashed curve), are drawn when the Hopf bifurcation occurs. This corresponds to $n=2.53$ with the other parameters given by (2) and $\bar{\tau}=7$ days. Periodic solutions appear with period of the oscillations about 33 days.

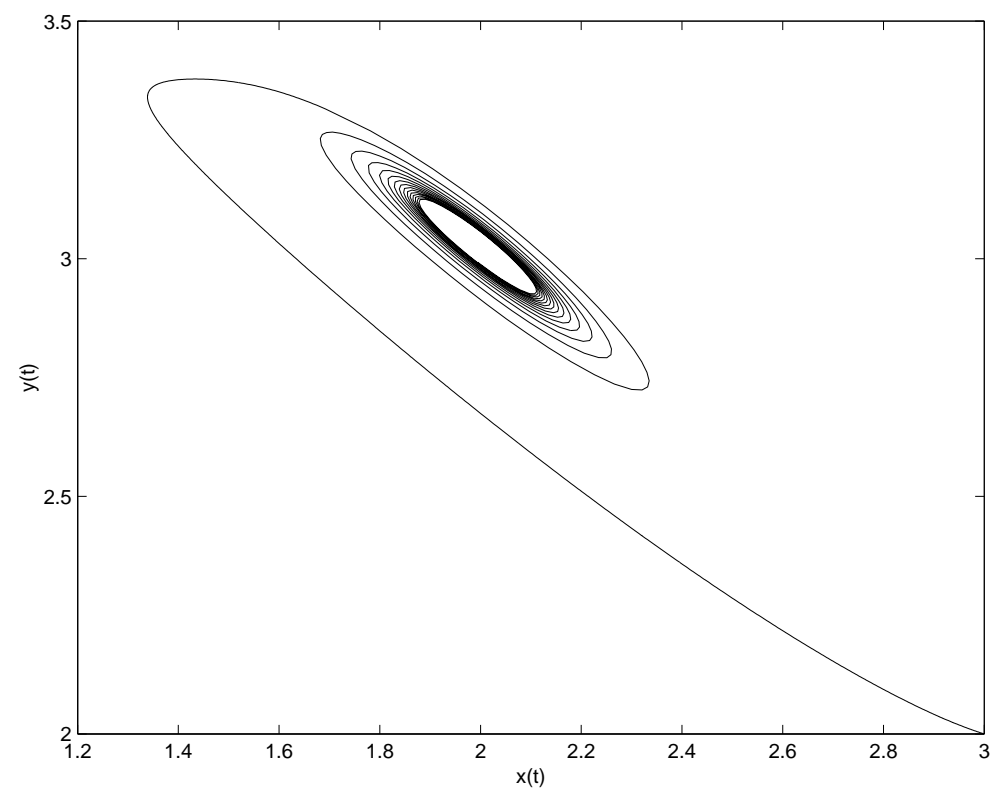

Figure 3: For the values used in Fig.2, the solutions are shown in the $(x, y)$-plane: the trajectories reach a limit cycle, surrounding the equilibrium. 


\section{Discussion}

Among the wide range of diseases affecting blood cells, periodic hematological diseases (Haurie et al. [14) are of main importance because of their intrinsic nature. These diseases are characterized by significant oscillations in the number of circulating cells, with periods ranging from weeks (19-21 days for cyclical neutropenia 14]) to months (30 to 100 days for chronic myelogenous leukemia 14) and amplitudes varying from normal to low levels or normal to high levels, depending on the cells types [14]. Because of their dynamic character, periodic hematological diseases offer an opportunity to understand some of the regulating processes involved in the production of hematopoietic cells, which are still not well-understood.

Some periodic hematological diseases involve only one type of blood cells, for example, red blood cells in periodic auto-immune hemolytic anemia (Bélair et al. 44) or platelets in cyclical thrombocytopenia (Santillan et al. 31]). In these cases, periods of the oscillations are usually between two and four times the bone marrow production delay. However, other periodic hematological diseases, such as cyclical neutropenia (Haurie et al. [14]) or chronic myelogenous leukemia (Fortin and Mackey 11), show oscillations in all of the circulating blood cells, i.e., white cells, red blood cells and platelets. These diseases involve oscillations with quite long periods (on the order of weeks to months). A destabilization of the pluripotential stem cell population (from which all of the mature blood cells types are derived) seems to be at the origin of these diseases.

We focus, in particularly, on chronic myelogenous leukemia (CML), a cancer of the white cells, resulting from the malignant transformation of a single pluripotential stem cell in the bone marrow (Pujo-Menjouet et al. [26]). As described in Morley et al. [24], oscillations can be observed in patients with CML, with the same period for white cells, red blood cells and platelets. This is called periodic chronic myelogenous leukemia (PCML). The period of the oscillations in PCML ranges from 30 to 100 days (Haurie et al. [14, Fortin and Mackey 11]), depending on patients. The difference between these periods and the average pluripotential cell cycle duration (between 1 to 4 days, as observed in mouses, see Mackey [18]) is still not well-understood.

Recently, to understand the dynamics of periodic chronic myelogenous leukemia, PujoMenjouet et al. [26] considered a model for the regulation of stem cell dynamics and investigated the influence of parameters in this stem cell model on the oscillations period when the model becomes unstable and starts to oscillate. In this paper, taking into account the fact that a cell cycle has two phases, that is, stem cells in process are either in a resting phase or actively proliferating, and assuming that cells divide at different ages, we proposed a system of differential equations with distributed delay to model the dynamics of hematopoietic stem cells. By constructing a Lyapunov functional, we gave conditions for the trivial equilibrium to be globally asymptotically stable. Local stability and Hopf bifurcation of the nontrivial equilibrium were studied, the existence of a Hopf bifurcation leading to the appearance of periodic solutions in this model, with a period around 30 days at the bifurcation.

Numerical simulations show that periodic solutions occur after the bifurcation, with periods increasing as the bifurcation parameter (the sensitivity $n$ ) increases. In Fig. 4, solutions oscillate around the equilibrium values with periods around 45 days. Moreover, amplitudes of the oscillations range from low values to normal values. The sensitivity is equal to $n=3$, that is the parameters are given by (2). This corresponds to values given by Mackey [16], values for which abnormal behavior (periodic) is usually observed in all circulating blood cells types.

When $n$ continues to increase, longer oscillations periods are observed with amplitudes varying from low values to high values (see Fig. 5). This situation characterizes periodic chronic myelogenous leukemia, with periods in the order of two months (70 days). 


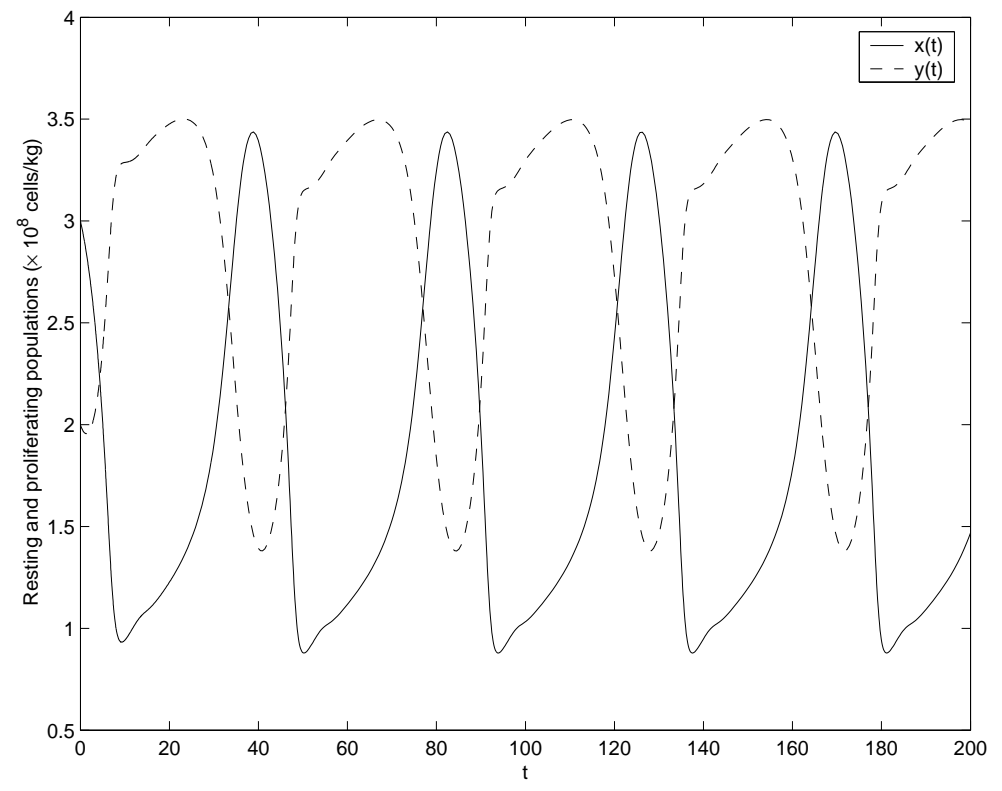

Figure 4: Solutions $x(t)$ (solid curve) and $y(t)$ (dashed curve) of system (12) oscillate with periods close to 45 days; the parameters are the same as in Fig 2, with $n=3$. The amplitudes of the oscillations range from low values to normal values.

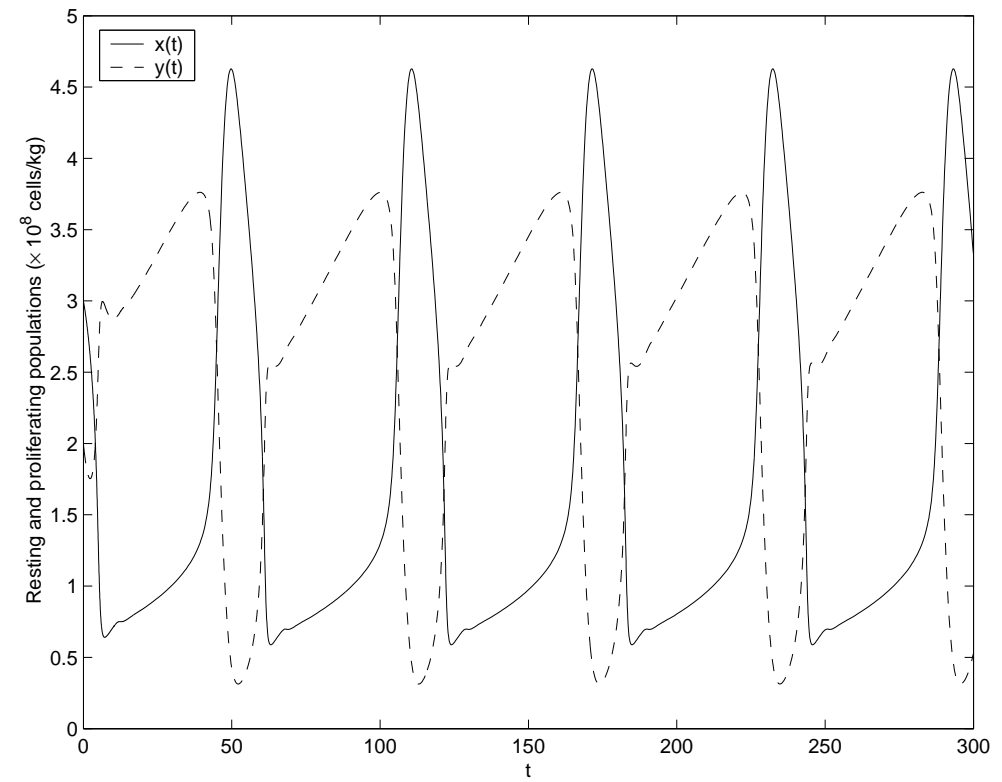

Figure 5: Solutions $x(t)$ (solid curve) and $y(t)$ (dashed curve) of system (12) oscillate with periods close to 70 days; the parameters are the same as in Fig 2, with $n=4$. The amplitudes of the oscillations range from low values to high values. 
Moreover, the oscillations observed in Fig. 1 and flook very much like relaxation oscillations. Experimental data from patients with PCML suggest that the shape of oscillations is of a relaxation oscillator type [11, 14]. Furthermore, Fowler and Mackey [12] showed that a model for hematopoiesis with a discrete delay may also exhibit relaxation oscillations. Therefore, it seems that not only periods and amplitudes of the oscillations correspond to the ones observed in PCML, but also the shape of the oscillations.

Numerical simulations demonstrated that long period oscillations in the circulating cells are possible in our model even with short duration cell cycles. Thus, we are able to characterize some hematological diseases, especially those which exhibit a periodic behavior of all the circulating blood cells.

\section{References}

[1] M. Adimy, F. Crauste and S. Ruan, Stability and Hopf bifurcation in a mathematical model of pluripotent stem cell dynamics, Nonlinear Analysis: Real World Applications (accepted).

[2] R. F. V. Anderson, Geometric and probabilistic stability criteria for delay systems, Math. Biosci. 105 (1991), 81-96.

[3] R. F. V. Anderson, Intrinsic parameters and stability of differential-delay equations, $J$. Math. Anal. Appl. 163 (1992), 184-199.

[4] J. Bélair, M. C. Mackey and J. M. Mahaffy, Age-structured and two-delay models for erythropoiesis, Math. Biosci. 128 (1995), 317-346.

[5] S. Bernard, J. Belair and M. C. Mackey, Sufficient conditions for stability of linear differential equations with distributed delay, Disc. Cont. Dyn. Syst. Ser. B 1 (2001), $233-256$.

[6] F. G. Boese, The stability chart for the linearized Cushing equation with a discrete delay and Gamma-distributed delays, J. Math. Anal. Appl. 140 (1989), 510-536.

[7] G. Bradford, B. Williams, R. Rossi and I. Bertoncello, Quiescence, cycling, and turnover in the primitive haematopoietic stem cell compartment, Exper. Hematol. 25 (1997), 445453.

[8] F. J. Burns and I. F. Tannock, On the existence of a $G_{0}$ phase in the cell cycle, Cell. Tissue Kinet. 19 (1970), 321-334.

[9] R. Crabb, J. Losson and M. C. Mackey, Dependence on initial conditions in non local PDE's and hereditary dynamical systems, Proc. Inter. Conf. Nonlin. Anal. 4, de Gruyter, Berlin, 1996, pp. 3125-3136.

[10] J. Dieudonné, Foundations of Modern Analysis, Academic Press, New-York, 1960.

[11] P. Fortin and M. C. Mackey, Periodic chronic myelogenous leukemia: Spectral analysis of blood cell counts and etiological implications, Brit. J. Haematol. 104 (1999), 336-345.

[12] A. C. Fowler and M. C. Mackey, Relaxation oscillations in a class of delay differential equations, SIAM J. Appl. Math. 63 (2002), 299-323.

[13] J. K. Hale, Theory of Functional Differential Equations, Springer-Verlag, New York, 1977. 
[14] C. Haurie, D. C. Dale and M. C. Mackey, Cyclical neutropenia and other periodic hematological diseases: A review of mechanisms and mathematical models, Blood 92 (1998), 2629-2640.

[15] Y. Kuang, Nonoccurrence of stability switching in systems of differential equations with distributed delays, Quart. Appl. Math. LII (3) (1994), 569-578.

[16] M. C. Mackey, Unified hypothesis of the origin of aplastic anaemia and periodic hematopoïesis, Blood 51 (1978), 941-956.

[17] M. C. Mackey, Dynamic hematological disorders of stem cell origin, in "Biophysical and Biochemical Information Transfer in Recognition", eds. by J. G. Vassileva-Popova and E. V. Jensen, Plenum Press, New York, 1979, pp. 373-409.

[18] M. C. Mackey, Cell kinetic status of haematopoietic stem cells, Cell Prolif. 34 (2001), 71-83.

[19] M. C. Mackey and L. Glass, From Clocks to Chaos: The Rhythms of Life, Princeton University Press, Princeton, 1988.

[20] M. C. Mackey and J. Milton, Feedback, delays, and the origins of blood cell dynamics, Comm. on Theor. Biol. 1 (1990), 299-327.

[21] M. C. Mackey and A. Rey, Bifurcations and travelling waves in a delayed partial differential equation, Chaos 2 (1992), 231-244.

[22] M. C. Mackey and R. Rudnicki, Global stability in a delayed partial differential equation describing cellular replication, J. Math. Biol. 33 (1994), 89-109.

[23] J. M. Mahaffy, J. Bélair and M. C. Mackey, Hematopoietic model with moving boundary condition and state dependent delay, J. Theor. Biol. 190 (1998), 135-146.

[24] A. A. Morley, A. G. Baikie and D. A. G. Galton, Cyclic leukocytosis as evidence for retention of normal homeostatic control in chronic granulocytic leukaemia, Lancet 2 (1967), 1320-1322.

[25] G. C. Nooney, Age distributions in dividing populations, Biophy. J. 7 (1967), 69-76.

[26] L. Pujo-Menjouet, S. Bernard and M. C. Mackey, Long period oscillations in a $G_{0}$ model of hematopoietic stem cells, preprint.

[27] L. Pujo-Menjouet and M. C. Mackey, Contribution to the study of periodic chronic myelogenous leukemia, C. R. Biologies 327 (2004), 235-244.

[28] S. I. Rubinow, A maturity time representation for cell populations, Biophy. J. 8 (1968), 1055-1073.

[29] S. I. Rubinow and J. L. Lebowitz, A mathematical model of neutrophil production and control in normal man, J. Math. Biol. 1 (1975), 187-225.

[30] L. Sachs, The molecular control of hemopoiesis and leukemia, C. R. Acad. Sci. Paris 316 (1993), 882-891.

[31] M. Santillan, J. Bélair, J. M. Mahaffy and M. C. Mackey, Regulation of platelet production: The normal response to perturbation and cyclical platelet disease, J. Theor. Biol. 206 (2000), 585-603. 
[32] L. F. Shampine and S. Thompson, Solving DDEs in Matlab, Appl. Numer. Math. 37 (2001), 441-458. http://www.radford.edu/ thompson/webddes/.

[33] E. Trucco, Mathematical models for cellular systems: The Von Foerster equation, Parts I and II, Bull. Math. Biophys. 27 (1965), 285-304; 449-470.

[34] E. Trucco, Some remarks on changing populations, J. Ferm. Technol. 44 (1966), 218226.

[35] G. F. Webb, Theory of nonlinear age-dependent population dynamics, Monographs and Textbooks in Pure and Applied Mathematics 89, Marcel Dekker Inc., New York, 1985. 